\title{
Localised neuronal migration disorder and intractable epilepsy: a prenatal vascular aetiology
}

David C Reutens, Samuel F Berkovic, Renate M Kalnins, Penny McKelvie, Michael M Saling, Gavin C A Fabinyi

Austin Hospital, Heidelberg, Australia, Department of Neurology

D C Reutens *

S F Berkovic*

Department of

Anatomical Pathology

R M Kalnins

P McKelvie

Department of Neuropsychology M M Salingt

Department of Neurosurgery

G C A Fabinyi

The University of

Melbourne,

Melbourne, Australia

Departments of

Medicine ${ }^{\star}$ and

Psychologyt

Correspondence to:

Dr Reutens, Department of Neurology, Austin Hospital, Heidelberg 3084, Australia.

Received 2 January 1992 and in revised form and in revised

Accepted 24 April 1992

Figure 1 A) Axial cranial MRI scan showing marked hypoplasia of the left occipital lobe; $B$ ) Coronal T1-weighted cranial MRI scan showing areas of ectopic grey matter in the subcortical white matter of the left posterior temporal lobe (arrow).

\begin{abstract}
Localised neuronal heterotopias are an increasingly recognised cause of intractable focal epilepsies. The aetiology of these circumscribed disorders of neuronal migration is often unknown although in some instances proximity to areas of prenatal infarction suggests that severe ischaemia was responsible. A patient is described with intractable complex partial seizures associated with heterotopic grey matter and cerebral hypoplasia confined to the territory of the left posterior cerebral artery; the left hippocampus was spared. Angiography showed a normal left anterior choroidal artery but a hypoplastic left posterior cerebral artery, implicating prenatal ischaemia without frank infarction as the aetiology of the malformation.
\end{abstract}

(F Neurol Neurosurg Psychiatry 1993;56:314-316)

Although circumscribed disturbances of neuronal migration have long been recognised in necropsy or surgical specimens, MRI has increased awareness of their importance as a cause of intractable focal epilepsies. ${ }^{12}$ In many cases the cause of the malformation is unknown. However, a vascular aetiology has been implicated when the disruption of histogenesis, most commonly polymicrogyria, lies adjacent to an area of putative infarction. ${ }^{3-5}$ We present evidence that nodular heterotopias may also result from ischaemia without frank infarction occurring early in gestation.

\section{Case report}

A 25 year old woman was referred for evaluation of intractable complex partial seizures. The perinatal period had been uneventful but between the ages of 18 and 24 months the patient had 7 brief febrile convulsions without focal features. Early development was normal. There was no family history of epilepsy.

At the age of 15, typical complex partial seizures started. They were characterised by an initial motionless stare, an unusual epigastric sensation, gustatory hallucinations, dystonic posturing of the right arm and automatisms. Secondarily generalised tonic-clonic seizures were infrequent. The seizures occurred at least weekly despite treatment with several anticonvulsants including carbamazepine, phenytoin and primidone.

Neurological examination revealed an incongruous, homonymous, peripheral right sided field defect. She was left handed, of average intellectual capacity and detailed neuropsychological assessment of visuoperceptual, language and material specific recent memory function was normal.

MRI showed that the left occipital lobe was considerably smaller than the right (fig 1A).
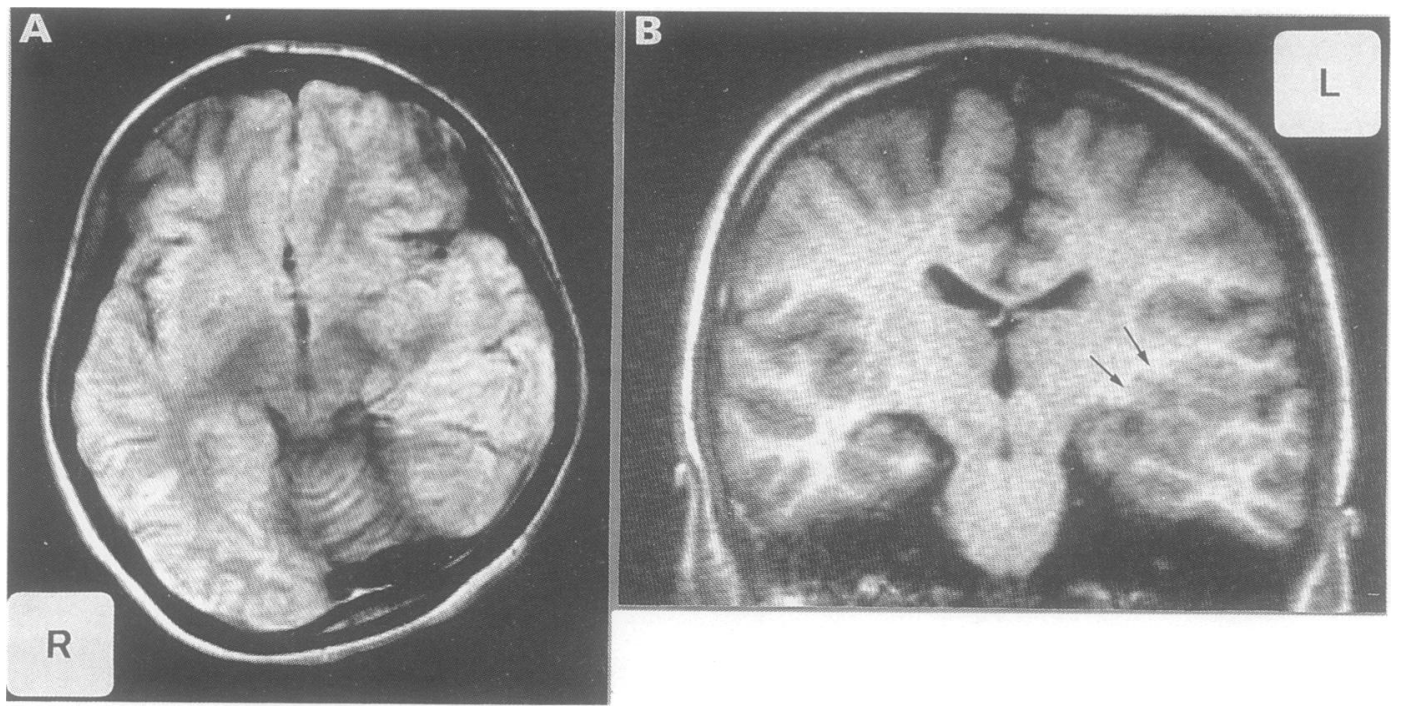


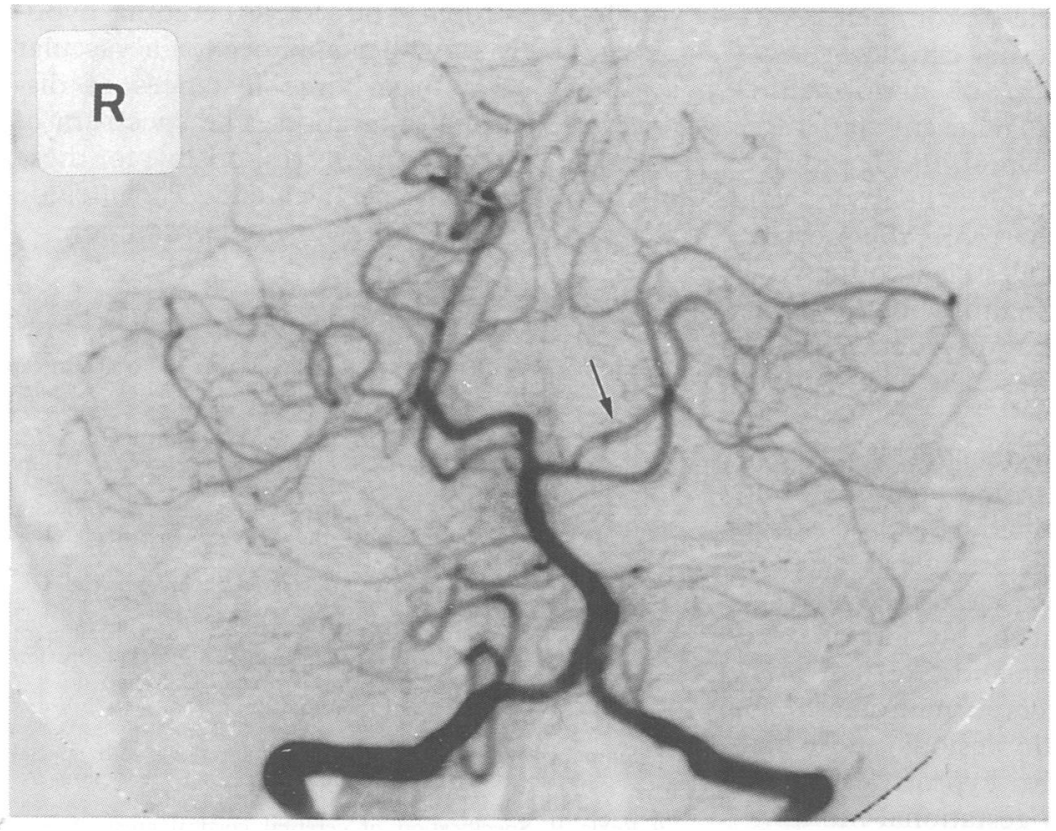

Figure 2 Right vertebral angiogram showing a hypoplastic left posterior cerebral artery (arrow) arising from the left superior cerebellar artery.

Areas of ectopic grey matter were present in the white matter of the left occipital and posterior left temporal lobes (fig 1B); the anterior quarter of the temporal lobe appeared unaffected. The left hippocampus appeared normal in size.

Figure 3 Section of the temporal lobe specimen. Normal inferomedial temporal neocortex is seen (A). A large mass of ectopic grey matter (*) and several smaller grey matter nodules $(\rightarrow)$ are present in the subcortical white matter. Luxol Fast Blue. Magnification $\times 5$.

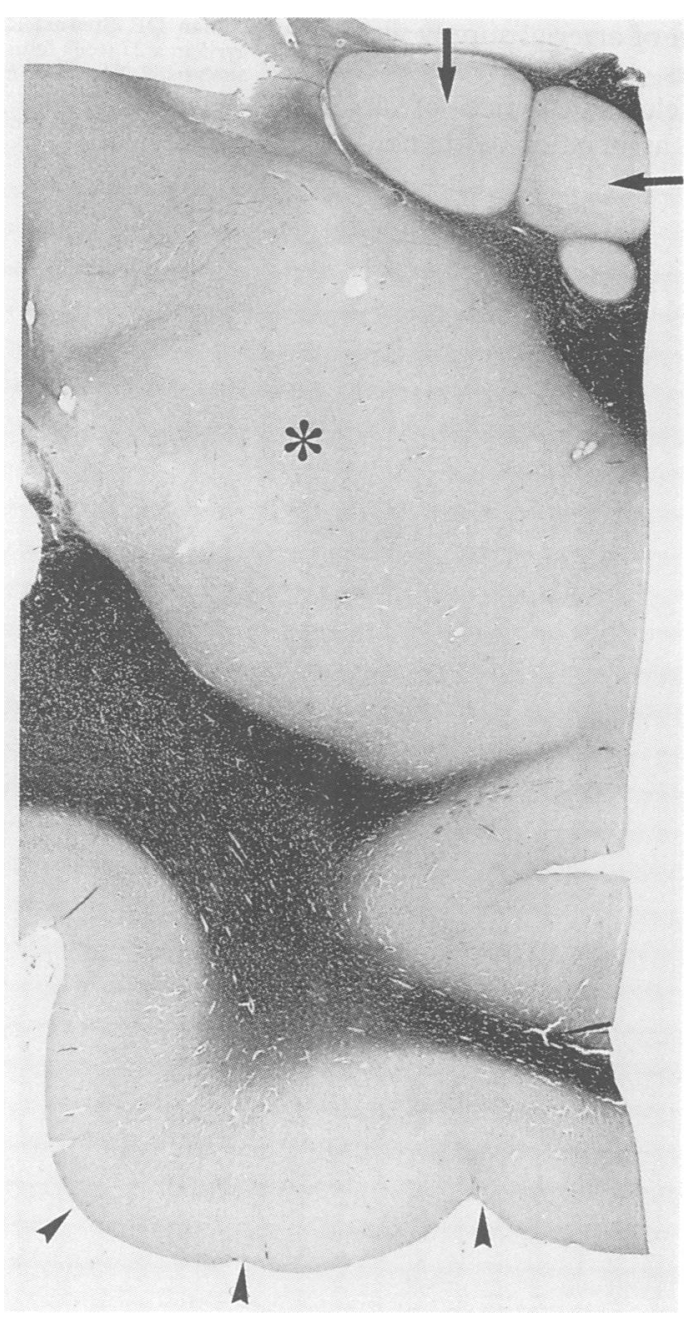

Interictal surface EEG recordings revealed an active epileptiform abnormality at $\mathrm{T} 3$ with normal background activity. Five ictal surface recordings and a recording employing sphenoidal electrodes showed rapid spike activity beginning at T3. An interictal blood flow study with ${ }^{99 \mathrm{~m}}$ Tc-HMPAO single photon emission computed tomography ${ }^{6}$ showed reduced left occipital perfusion in the region of the structural abnormality. Marked hyperperfusion in the left temporal region was present in the ictal study.

At angiography, a hypoplastic left posterior cerebral artery was seen after vertebral and left internal carotid artery injections. It arose from the ipsilateral superior cerebellar artery (fig 2). The left anterior choroidal artery was normal. A left internal carotid artery amytal test produced a brief period of anarthria followed by normal naming, reading, sentence repetition and preserved verbal specific memory.

Left temporal lobectomy was performed; the neocortical margin of resection was $6.5 \mathrm{~cm}$ from the temporal tip. The mesial temporal structures were also removed. Microscopic examination of the neocortex of the temporal pole and lateral temporal lobe revealed normal vertical and horizontal lamination. In the white matter of the temporal lobe, ectopic masses of grey matter composed of randomly organised large and medium sized neurons with associated oligodendrocytes and astrocytes were present (fig 3). The histology of the anterior hippocampus was normal with no evidence of pyramidal cell loss, gliosis or neuronal heterotopia.

In the eighteen months following temporal lobectomy, she has experienced no complex partial seizures and only rare auras.

\section{Discussion}

During fetal development, radial glial fibres span the telencephalon, guiding the migration of neurons from the ventricular and subventricular areas to their destinations in the cerebral cortex. Destruction of these fibres plays an important role in the pathogenesis of neuronal migration disorders. The majority of neurons migrate between 7 and 16 weeks gestational age making it likely that the developmental insult occurred in the early fetal period in this patient. $^{7-9}$ Absence of glial scarring is further evidence for an early gestational lesion; during this period the cell bodies of astrocytes are restricted to the periventricular region. ${ }^{10}$

We propose that the aetiology of the neuronal migration abnormality in this patient was intrauterine ischaemia in the territory of the left posterior cerebral artery. The artery was hypoplastic with an anomalous origin. The salient abnormalities in our patient, left occipital hypoplasia and occipitotemporal grey matter heterotopia, were limited to the artery's zone of perfusion. In keeping with the fetal pattern of arterial supply, hippocampal histology and thalamic morphology on MRI were unaffected. The developing hippocampus and diencephalon are supplied by the anterior and 
posterior choroidal arteries, prominent branches of the primitive internal carotid artery. ${ }^{11}$ The peculiar distribution of abnormalities makes a primary vascular event far more plausible than a patchy, multifocal pathogenetic process affecting both neuronal and vascular structures. Furthermore, the presence of normal major cerebral vessels in other cases with substantial abnormalities of neuronal migration and peroxisomal disorders supports the case for a primary vascular event in this patient. $^{12}$

Intrauterine cerebral ischaemia is a recognised cause of disordered neuronal migration. In most previously described cases, the ischaemia has been severe. For example, Dekaban, ${ }^{3}$ Levine et $a l^{4}$ and Ferrer ${ }^{5}$ described areas of polymicrogyria located at the borders of extensive porencephalic defects and Norman ${ }^{13}$ described heterotopic clusters of neuroblasts and bilateral encephaloclastic lesions in a fetal brain following a putative hyproxic-ischaemic insult at about the third gestational month.

Can abnormal histogenesis occur with ischaemia insufficient to produce frank infarction? Our case supports this possibility. Barth and van der Harten ${ }^{14}$ describe the product of parabiotic twin pregnancy with focal cerebral hypoplasia, microgyria and nodular heterotopias in the territories of bilateral hypoplastic posterior cerebral arteries. Richman et $\mathrm{al}^{15}$ and McBride and Kemper ${ }^{16}$ have also described cases of microgyria restricted to a vascular territory and unassociated with cavitation; however, the description of arterial supply was incomplete in both cases.

Our case provides clear evidence of an arterial abnormality in association with neu- ronal heterotopia and focal cerebral hypoplasia. It gives further support for a vascular aetiology in at least some instances of disordered neuronal migration. The spectrum of pathogenetic mechanisms responsible for these malformations has been extended to include reduced perfusion without frank infarction.

1 Osborn RE, Byrd SE, Naidich TP, et al. MR imaging of neuronal migrational disorders. AfNR 1988;9:1101-6.

2 Kuzniecky R, Berkovic S, Andermann F, et al. Focal cortical myoclonus and rolandic dysplasia: clarification by mag netic resonance imaging. Ann Neurol 1988;23:317-25.

3 Dekaban A. Large defects in cerebral hemispheres associated with cortical dysgenesis. $\mathcal{f}$ Neuropath Exp Neurol 1965;24:512-30.

4 Levine DN, Fisher MA, Caviness VS. Porencephaly with microgyria: a pathologic study. Acta Neuropathol (Berl) 1974:29:99-113.

5 Ferrer I. A Golgi analysis of unlayered polymicrogyria. Acta Neuropathol (Berl) 1984;65:68-76.

6 Rowe CC, Berkovic SF, Sia STB, et al. Localisation of epileptic foci with postictal single photon emission computed tomography. Ann Neurol 1989;26:660-8.

7 Barth PG. Disorders of neuronal migration. Can 7 Neurol Sci 1987;14:1-16.

8 Sidman RI, Rakic P. Neuronal migration, with special reference to developing human brain: a review. Brain Res 1973;62:1-35.

9 Rakic P. Specification of cerebral cortical areas. Science 1988:241:170-6.

10 Fujita S. Cytogenesis and pathology of neuroglia and microglia. Path Res Pract 1980;168:271-8.

11 Padget DH. The development of the cranial arteries in the human embryo. Carnegie Inst of Wash Contrib Embryol 1948;22:205-61.

12 Evrard P, Caviness VS, Prats-Vinas J, Lyon G. The mechanism of arrest of neuronal migration in the Zellweger malformation: an hypothesis based upon cytoarchitecger malformation: an hypothesis based upon cytoarchitec-
tonic analysis. Acta Neuropathol (Berl) 1978;41:109-17.

tonic analysis. Acta Neuropathol (Berl) 1978;41:109-17.
13 Norman MG. Bilateral encephaloclastic lesions in a 26 week gestation fetus: effect on neuroblast migration. Can $f$

Neurol Sci $1980 ; 7: 191-4$.
14 Barth PG, van der Harten JJ. Parabiotic twin syndrome with topical isocortical disruption and gastroschisis. Acta Neuropathol (Berl) 1985;67:345-9.

15 Richman DP, Stewart RM, Caviness VS. Cerebral microgyria in a 27 week fetus: an architectonic and topographic analysis. $\mathcal{F}$ Neuropath Exp Neurol 1974;33:374-84.

16 McBride MC, Kemper TL. Pathogenesis of four-layered microgyric cortex in man. Acta Neuropathol (Berl) 